\title{
Unusual behavior of sound velocity of a Bose gas in an optical superlattice at quasi-one-dimension
}

\author{
Lei Chen, ${ }^{1}$ Zhu Chen, ${ }^{2} \mathrm{Wu} \mathrm{Li},{ }^{1}$ Zhidong Zhang, ${ }^{1}$ and Zhaoxin Liang ${ }^{1, *}$ \\ ${ }^{1}$ Shenyang National Laboratory for Materials Science, Institute of Metal Research, \\ Chinese Academy of Sciences, Wenhua Road 72, Shenyang 110016, China \\ ${ }^{2}$ National Key Laboratory of Science and Technology on Computational Physics, \\ Institute of Applied Physics and Computational Mathematics, Beijing 100088, China
}

(Dated: July 30, 2021)

\begin{abstract}
A Bose gas trapped in a one-dimensional optical superlattice has emerged as a novel superfluid characterized by tunable lattice topologies and tailored band structures. In this work, we focus on the propagation of sound in such a novel system and have found new features on sound velocity, which arises from the interplay between the two lattices with different periodicity and is not present in the case of a condensate in a monochromatic optical lattice. Particularly, this is the first time that the sound velocity is found to first increase and then decrease as the superlattice strength increases even at one dimension. Such unusual behavior can be analytically understood in terms of the competition between the decreasing compressibility and the increasing effective mass due to the increasing superlattice strength. This result suggests a new route to engineer the sound velocity by manipulating the superlattice's parameters. All the calculations based on the mean-field theory are justified by checking the exponent $\gamma$ of the off-diagonal one-body density matrix that is much smaller than 1. Finally, the conditions for possible experimental realization of our scenario are also discussed.

PACS numbers: 67.85.-d,67.85.De,67.85.Hj,03.75.Kk
\end{abstract}

\section{INTRODUCTION}

Sound propagation plays a fundamental role in understanding the superfluid behavior [1-6]. Ever since the first achievement of Bose-Einstein condensate (BEC) in atomic gases, the sound velocity has been one of the first things to be studied theoretically [1] and experimentally [7-11] on a BEC in the presence of harmonic traps [1215], optical lattices [16-27] and disorder [28, 29], etc. More recently, the studies on the sound velocity have been renewed, which are much more in line with its important applications in quantum simulations involving superfluid [30-32]. For instance, in recent proposals concerning the preparations of many-body states and nonequilibrium quantum phases based on engineering a superfluid reservoir [33-36], the sound velocity acts as a key parameter in determining the system-bath coupling. The control of sound velocity of a superfluid, such as by using various carefully configured external traps [1629], therefore constitutes an important ingredient of the reservoir engineering.

In this work, we study the sound velocity of a BEC loaded in an optical superlattice (OSL). The motivation behind this work ties closely to the recent progress in engineering novel optical lattices in atomic setups, which is highlighted by the advances in the superlattice technology. Compared with conventional monochromatic optical lattice (OL), an OSL is characterized by several distinguishing features [37-42] that we have: (i) an additional OL of $d_{2}$ periodicity superimposed on a fundamental OL

\footnotetext{
* Corresponding author: zhxliang@gmail.com
}

of $d_{1}$ periodicity, and (ii) a complete control of the relative phase between two lattices. In the early days of OSL experiments, the most of investigations have either focused on the static and dynamical properties of the condensate like the coherence $[38,43]$ or employed two non-commensurate lattices to simulate disorder quantum system [44]. More recently, emphasis has been shifted to a BEC loaded in an OSL that has emerged as a novel kind of superfluid with tunable real-space lattice topologies (sub-structures within the unit cell) and band structures [37]. In more details, loading cold atoms in an OSL with configurable structures, together with non-equilibrium control of lattice intensity and phases, has led to, for example, the observation of the Zak phase connected to the topology of the band structure at one dimension (1D) $[45,46]$, as well as opened a new avenue in atomic implementations [47, 48], such as the simulations of Dirac fermions in interacting relativistic field theories [49], and the controlled coherent transport of atomic wave packets [50] or charge pumping [51]. Along this research line, we expect that an OSL can lead to new features on the sound velocity not present in the conventional monochromatic OL.

To further motivate our investigation on the sound velocity of a BEC in an OSL, we first recall that, in an OL, the behavior of sound velocity of a BEC is believed to be determined by the interplay among three parameters: the strength of the optical lattice, $V_{1}$; the interaction between atoms, $c$; and the lattice dimension, $\mathrm{D}(\mathrm{D}=1,2$, and 3). Theoretical studies have shown: (i) when $\mathrm{D}=1$, the sound velocity always decreases monotonically with increasing $V_{1}$ [18-24]. (ii) for $\mathrm{D}=2$ and 3, when $c$ exceeds a critical value, the sound speed first increases to 
a maximum value and then decreases with increasing $V_{1}$ $[25,26]$. When $\mathrm{D}=3$, the sound velocity can even oscillate with respect to $V_{1}[26]$. Such rich behavior of sound velocity in an OL can be understood in terms of compressibility $\kappa$ and effective mass $m^{*}$ as $c_{s}=\sqrt{1 / \kappa m^{*}}$. So far all the investigations suggest that the sound velocity of an optically-trapped 1D BEC always decreases monotonically with increasing the lattice strength. Considering that both $m^{*}$ and $\kappa$ may be strongly affected by the topological structure of a 1D OSL due to the introduction of additional freedom, we expect that a different behavior of sound velocity of a BEC in an OSL may occur.

In this paper, we are then motivated to launch systematic studies on an interacting BEC loaded into an OSL at quasi-1D using both the analytical and numerical methods and show that these systems display the nontrivial properties of the sound velocity, which share the same physical origins of sound velocity in 2D (3D) BEC in terms of $c_{s}=\sqrt{1 / \kappa m^{*}}$. For example, our analytical results in the limit of weak potential display that both the relative phase and the strength of the two lattices that form a 1D OSL can significantly influence the sound velocity of a BEC. In particular, a surprise indeed arises that the sound velocity can first increase and then decrease when the OSL strength increases even at 1D, which presents a contrast to a quasi-1D BEC in a 1D monochromatic OL where the sound velocity always decrease with increasing lattice strength. Such behavior of sound velocity are then verified by the numerical results and can be understood in terms of the competition between the compressibility $\kappa$ and the effective mass $m^{*}$. In addition, our further numerical results show that the sound velocity exhibits very rich behavior with respect to various choices of OSL parameters. Our study suggests a new route to engineer the sound velocity by loading a superfluid in an OSL.

The paper is organized as follows. In Section II we derive the effective model for a quasi-1D BEC in an OSL. In Section III we study the sound propagation and its velocity of the model system, using both analytical and numerical approaches, in different parameter regimes, and finally in Section IV we summarize our results and give an outlook.

\section{A QUASI-1D BEC IN OPTICAL SUPERLATTICE POTENTIALS}

\section{A. Effective model}

We consider a bulk BEC (see Fig. 1a) trapped in an OSL of $V_{\mathrm{OSL}}(x)$ along the $x$-direction, whereas the model system is uniform in the $y$ - and the $z$-directions [52]. The OSL of $V_{\mathrm{OSL}}(x)$ is made of a fundamental lattice in the $x$ direction with the spatial period $d$ denoted as the primary lattice, and an additional lattice with a period $d / 2$ named by the secondary lattice. The corresponding expression for $V_{\mathrm{OSL}}(x)$ is

$$
V_{\mathrm{OSL}}(x)=V_{1} \sin ^{2}\left(k_{L} x\right)+V_{2} \sin ^{2}\left(2 k_{L} x+\theta\right) .
$$

Here, $k_{L}=\pi / d(\lambda=2 d)$ is the wave vector (wave length) of the laser light creating the OSL and $\theta$ is the relative phase between the two constituting lattices characterized by the lattice strength $V_{1(2)}$ in unit of the recoil energy of $E_{R}=\hbar^{2} k_{L}^{2} / 2 m$. Such an OSL in equation (2.1) has been experimentally realized for quantum gases in references [39, 40] with $V_{1(2)}$ depending on the internal state of an atom (see Fig. 1b for a typical scheme of OSL in bosonic atomic gases). For the condensate density along the transverse directions in uniform, the freedom along $y$ and $z$-directions decouples from the $x$-direction, leading to the realization of a quasi-1D geometry $[1,2,26]$,

$$
H-\mu N=\frac{1}{d} \int d x \Psi^{*}\left[-\frac{\hbar^{2}}{2 m} \frac{\partial^{2}}{\partial x^{2}}+V_{\mathrm{OSL}}(x)+\frac{g n_{0} d}{2}|\Psi|^{2}\right] \Psi,
$$

where $\Psi(x)$ is the condensate wave function satisfying the normalizing condition $1=\int_{0}^{d} d x \Psi^{*}(x) \Psi(x)$ with $m$ being the atomic mass, $\mu$ the chemical potential, $N$ the atom number in condensate, $n_{0}$ the $3 \mathrm{D}$ average density and $g n_{0}$ capturing the role of interactions in the system. In general, the presence of external confinements will affect the collision property between two particles and the $g$ can deviate substantially from the coupling constant $g_{3 D}$ of a free 3D Bose gas $\left(g_{3 D}=4 \pi \hbar^{2} a_{3 D} / m\right.$ with $a_{3 D}$ being the 3D scattering length). The derivation of $g$ in the presence of external potentials is highly nontrivial [53]. Here, following reference [19], we limit ourself to the parameter regime $g=a_{3 D}$ and the typical values of $g n_{0} d / E_{R}$ in experiments ranges from 0.02 to 1 .

Next, we rescale equation (2.2) by introducing dimensionless variables, $x^{\prime}=2 k_{L} x, t^{\prime}=t / 8 E_{R}, \psi=\Psi / \sqrt{n_{0}}$, $\phi=2 \theta+\pi, v_{1(2)}=V_{1(2)} / 16 E_{R}, c=g n_{0} d / 8 E_{R}$, and $\mu / 8 E_{R} \rightarrow \mu$. Finally, we arrive at an effective 1D Hamiltonian describing our model system [54-57],

$$
\begin{aligned}
H_{1 D}=\frac{1}{2 \pi} \int_{-\pi}^{\pi} d x \psi^{*}(x)[ & -\frac{1}{2} \frac{\partial^{2}}{\partial x^{2}}+V_{\mathrm{OSL}}(x) \\
& \left.+\frac{c}{2}|\psi(x)|^{2}-\mu\right] \psi(x)(2.3)
\end{aligned}
$$

with

$$
V_{\mathrm{OSL}}(x)=V_{1} \cos (x)+V_{2} \cos (2 x+\theta) .
$$

The Hamiltonian (2.3) describes a quasi-1D BEC in an OSL at the mean-field level. The corresponding physics is determined by the effective interatomic interaction $c$, and the parameters characterizing an OSL of $V_{\mathrm{OSL}}(x)\left(V_{1(2)}\right.$ and $\phi)$. In order to visualize the superlattice potential $V_{\mathrm{OSL}}(x)$ and highlight the role of the relative phase $\phi$ in affecting the system, we have plotted $V_{\mathrm{OSL}}(x)$ (see Fig. 1c) as a function of the relative phase $\phi$. It is obvious from the plot that, when $\phi$ increases from 0 to $\pi$ with fixed lattice intensities $v_{1(2)}$, the OSL potential becomes 

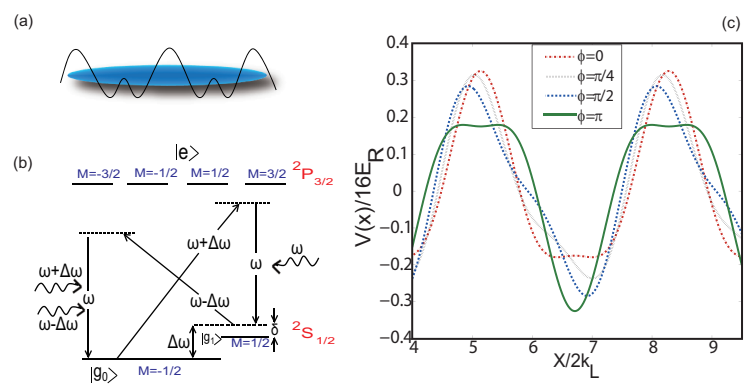

FIG. 1. (Color online) (a) Quasi-1D BEC trapped in an optical superlattice. (b) A four-photon optical superlattice for ${ }^{87} \mathrm{Rb}$ atoms: the two states $\left|F=1, M_{F}=-1\right\rangle$ and $\left|F=1, M_{F}=0\right\rangle$ serves as $\left|g_{0}\right\rangle$ and $\left|g_{1}\right\rangle$ and the $5 P_{3 / 2}$ excited state manifold as $|e\rangle$. The supperlattice depth $V_{2}$ is given by $(\hbar / 2 \delta) \Omega_{\text {eff }}^{+} \Omega_{\text {eff }}^{-}$with $\Omega_{\text {eff }}^{+}=\Omega_{\mathrm{g}_{0}, \mathrm{e}}^{+} \Omega_{\mathrm{e}, \mathrm{g}_{1}}^{0} / 2 \Delta$ and $\Omega_{\mathrm{eff}}^{-}=\Omega_{\mathrm{g}_{1}, \mathrm{e}}^{-} \Omega_{\mathrm{e}, \mathrm{g}_{0}}^{0} / 2 \Delta$. Here, $\Omega_{\mathrm{g}_{0}, \mathrm{e}}^{+}$and $\Omega_{\mathrm{e}, \mathrm{g}_{0}}^{0}$ are the Rabi frequencies for the transitions $\left|g_{0}\right\rangle \leftrightarrow|e\rangle$ and $|e\rangle \leftrightarrow\left|g_{1}\right\rangle$ driven by the light fields with frequency $\omega+\Delta \omega$ and $\omega$, respectively [3942]. Furthermore, we assume that the condition of $\Delta \gg \Delta \omega$ can be fulfilled for all three optical frequencies. (c) Typical optical superlattice structure with different values $\phi=2 \theta+\pi$. The parameters are given as $V_{1}=v_{1} \times 16 E_{R}=4 E_{R}$ and $V_{2}=v_{1} \times E_{R}=1.2 E_{R}$, respectively.

more squeezed, which can result in enhanced repulsive interatomic interaction.

The quasi-1D condensate wave function $\psi(x)$ and the chemical potential $\mu$ in equation (2.3) are determined from the corresponding Gross-Piteavskii equation (GPE) reading $[1]$

$$
\left[-\frac{1}{2} \frac{\partial^{2}}{\partial x^{2}}+V_{\mathrm{OSL}}(x)+c|\psi(x)|^{2}\right] \psi(x)=\mu \psi(x) .
$$

Note that for OSL in equation (2.4), the Hamiltonian (2.3) is still periodic in the $x$-direction, so the condensate function $\psi(x)$ is represented by a Bloch state satisfying

$$
\psi(x)=e^{i k x} \varphi_{k}(x)
$$

with

$$
\varphi_{k}(x)=\sum_{m} a_{m} e^{i m x}=\sum_{m}\left(\left|a_{m}\right| e^{i \phi_{m}}\right) e^{i m x},
$$

where $k$ is the Bloch wave vector, $\varphi_{k}$ is a periodic function with the periodicity of $2 \pi$, and $a_{m}$ is the expansion coefficient. It is worth mentioning that the concept of Bloch wave function is originally introduced for linear periodic systems $[58,59]$, which can also be extended to weakly nonlinear periodic quantum systems. Hence, it is reasonably expected that the ground state of equation (2.5) should occur to the state of $\mathrm{k}=0$ in equation (2.6).

An important feature of the Bloch state $\psi(x)$ in equation (2.6) is that the expansion coefficient $a_{m}$ in equation (2.7) is complex with $a_{m}=\left|a_{m}\right| e^{i \phi_{m}}$. This feature immediately distinguishes the Bloch state in an OSL from the one in an OL, where $a_{m}$ must be real. The origin of such difference is that the conventional OL of $V_{\mathrm{OL}}(x)$ has a parity symmetry $\left(V_{\mathrm{OL}}(-x)=V_{\mathrm{OL}}(x)\right)$, which dictates the imaginary part of $a_{m}$ to varnish, whereas an OSL of $V_{\mathrm{OSL}}(x)$ usually does not have such symmetry, i.e. $V_{\mathrm{OSL}}(-x) \neq V_{\mathrm{OSL}}(x)$ for $\phi \neq 0$. In addition, the superposition of an additional lattice gives rise to the emergence of substructures in each lattice unit cell in the real space, which can result in substantial modification of the band structure. For example, for suitable choices of $\phi$ and $V_{1(2)}$ as have been pointed out in references [39-42], the dispersion relation in the region between the first and second Bloch band can be tuned to be linear, a feature reminiscent of relativistic particles.

\section{B. Quantum fluctuations and regimes of validity of the mean-field theory}

For an optically trapped BEC, the phase fluctuations due to the periodic potential will be inevitable to reduce the coherence of a BEC, leading to a quantum phase transition from the superfluid to the Mott insulator. In this work, we limit ourselves to the parameter regimes, where the mean-field theory based on GP equation is always valid. Because the phase fluctuations induced by OL depend on explicitly on the geometry of the model system, we here emphasize the geometry of a BEC considered here as follows: along the $x$-direction, the atoms can feel an OSL, in contrast, the model system is uniform in the transverse direction. We now proceed to present two approaches for justifying the validity of the meanfield theory.

Generally speaking, the mean-field theory based on GP equation is reliable upon the quantum depletion of $N_{e x}$ being small relative to the total atom number of $N$, i.e. $N_{e x} \ll N$. Hence, we posteriori justify the mean-field theory by computing the quantum depletion of $N_{e x} / N$. After that, we choose the safe parameter regimes by letting $N_{e x} / N \ll 1$. Following reference [19], we can obtain the analytical expression of quantum depletion of the condensate as follows,

$\frac{N_{e x}}{N}=2 \frac{a_{3 D}}{\sqrt{2 \pi} \sigma}\left[\frac{1}{2}-\frac{\sqrt{b}}{\pi}+\frac{b}{2}-\arctan (\sqrt{b})(1+b) / \sqrt{\pi}\right]$,

with

$$
\begin{aligned}
b & =\frac{2 \sqrt{2}}{\sqrt{\pi^{3}}} \frac{m}{m^{*}} \frac{\sigma}{d} \frac{E_{R}}{g n_{0} d}, \\
\sigma & =\frac{d}{2 \pi} \frac{1+1 / 16 \sqrt{v_{1}+v_{2} \cos [2 \phi]}}{\left(v_{1}+v_{2} \cos [2 \phi]\right)^{1 / 4}}
\end{aligned}
$$

Based on equation (2.8), two conclusions are immediately made: (i) equation (2.8) can recover the well-known $3 \mathrm{D}$ result of $N_{e x} / N=(8 / 3 \sqrt{\pi}) \sqrt{m^{*} / m}\left(n a^{\prime 3}\right)^{1 / 2}$ with $a^{\prime}=a_{3 D} d / \sqrt{2 \pi} \sigma$ in teh limit of weak potential; (ii) then, and the validity regimes of the mean-field theory can be 
further identified by the conditions [19] as

$$
N_{e x} / N \approx m^{*} c_{s} d /\left(2 \pi \hbar N_{t o t}\right) \log \left(4 N_{\perp} / \pi\right) \ll 1
$$

with $N_{\perp}$ being the number of lattice wells along $x$ direction. As emphasized in reference [19], the mean-field theory of equation (2.2) is still valid at $V_{1}=v_{1} \times 16 E_{R}=$ $20 E_{R}$ under the conditions of the typical experimental parameters $g n_{0} d=0.2 E_{R}$ and $N_{\perp}=200$.

On the other hand, we can justify the mean-field theory by checking the asymptotical values of the off-diagonal one-body density matrix at large distance as follows $[2$, $19]$

$$
n^{(1)}\left(\left|\mathbf{r}-\mathbf{r}^{\prime}\right|\right) \rightarrow\left|\mathbf{r}-\mathbf{r}^{\prime}\right|^{-\gamma}
$$

with $\gamma=m^{*} c_{s} d /\left(2 \pi \hbar N_{w e l l}\right)$. Here, $N_{\text {well }}$ is the number of particles per well. We have checked that the exponent $\gamma$ is much smaller than 1 in our parameter regimes, which means that the coherence survives at large distances and the application of the mean-field theory based on GP equation is justified.

\section{SOUND VELOCITY}

The previous section sets the stage for our study on the sound propagation in a BEC trapped in an OSL. In this section, we outline both the analytical and numerical approaches to the sound velocity of a BEC.

\section{A. Sound velocity in an optical lattice}

For an optically trapped BEC, the sound propagation and its velocity can be viewed from two different perspectives [26]. The first perspective establishes the sound propagation in a $\mathrm{BEC}$ as a long-wavelength response to an external perturbation, which thus connects the sound velocity to the microscopic excitations of a $\mathrm{BEC}[1,2]$. In an OSL, the sound velocity of a quasi-1D BEC is given by

$$
c_{s}=\lim _{p \rightarrow 0} \frac{\epsilon_{p}}{\hbar p}
$$

where $p$ and $\epsilon_{p}$ are the quasi-momentum of the probe and the energy of the excitation, respectively. In equation (3.1), the effects of the lattice is encoded in the energy spectrum $\epsilon_{p}$.

In the second perspective, on the other hand, the sound velocity $c_{s}$ is intimately related to the superfluidity of a BEC and its macroscopic dynamics, where the sound velocity can be written as $[3,4,26]$

$$
c_{s}=\sqrt{\frac{1}{\kappa m^{*}}} .
$$

Here, the compressibility $\kappa$ and the effective mass $m^{*}$, are defined as, respectively,

$$
\frac{1}{m^{*}}=\lim _{k \rightarrow 0} \frac{d^{2} E_{k}}{\hbar^{2} d k^{2}}
$$

and

$$
\kappa^{-1}=n_{0} \frac{\partial \mu}{\partial n_{0}}
$$

with $n_{0}$ being the $3 \mathrm{D}$ condensate density, $E_{k}$ the average energy of the system obtained by plugging equation (2.6) into equation (2.3) and $\mu=\partial E_{k} / \partial n_{0}$ the chemical potential. The effect of an OSL is thus encoded in the increased effective mass $m^{*}$ along the lattice direction, and the enhanced interatomic interaction which manifests itself in the decreased compressibility $\kappa$. The two definitions on the sound velocity (Eqs. (3.1) and (3.2)) have been proved equivalent $[3,4,19]$ for an optically trapped BEC.

\section{B. Sound velocity in an OSL: perturbation approach}

We now analyze the effect of an OSL of $V_{\mathrm{OSL}}(x)$ $\left(V_{2} \neq 0\right.$ in Eq. (2.4)) on the sound velocity of a quasi-1D BEC. In this section, we will assume the strengh of $v_{2}$ is weak, and then consider two regimes associated with the strengh of $v_{1}$ : in the first regime, hereafter referred to as the weak superlattice regime, $v_{1}$ is comparable with $v_{2}$ and are both small (say, $v_{1} \sim v_{2} \sim \lambda$ with $\lambda$ being introduced to label the order of perturbation and finally set to be one); in the second regime, hereafter referred to as the tight-binding regime, $v_{1}$ is much stronger than $v_{2}$, i.e. $v_{1} \gg v_{2}$ (we assume $v_{1}$ is still sufficient to maintain the coherence of BEC over several optical wells). Subsequently, we will derive analytically the sound velocity in the two regimes, respectively.

\section{Weak superlattice regime}

In the weak superlattice regime with $v_{1} \sim v_{2} \sim \lambda$, the whole superlattice potential $V_{\mathrm{OSL}}(x)$ in Hamiltonian (2.3) can be treated as a perturbation to a unperturbed system consisting of a homogeneous quasi-1D BEC. In this case, we solve the GPE (2.5) by developing a perturbation expansion to the condensate wave function $\psi(x)$ up to the second order of the small parameters $v_{1} \sim v_{2} \sim \lambda$, i.e.

$$
\psi(x)=\psi^{(0)}(x)+\psi^{(1)}(x)+\psi^{(2)}(x)+o\left(\lambda^{3}\right) .
$$

Direct application of the perturbation procedures [26] to the GPE (2.5) order by order thus yields $\psi^{(0)}(x)=1$, as 
it is should be, and

$$
\begin{aligned}
\psi^{(1)} & =-\frac{1+k}{1+c-k^{2}} \frac{v_{2}}{4} e^{-2 i x-i \phi}-\frac{2 k-1}{4 k^{2}-4 c-1} v_{1} e^{-i x} \\
& +\frac{2 k+1}{4 k^{2}-4 c-1} v_{1} e^{i x}-\frac{1-k}{1+c-k^{2}} \frac{v_{2}}{4} e^{2 i x+i \phi}
\end{aligned}
$$

as well as

$$
\begin{aligned}
\psi^{(2)} & =\left[\frac{2+2 k}{4+4 c-4 k^{2}} C+\frac{c}{4+4 c-4 k^{2}}(D-C)\right] v_{1}^{2} e^{-2 i x} \\
& -\left[\frac{2(1+2 k+2 c)}{1+4 c-4 k^{2}} B^{*}+\frac{4 c}{1+4 c-4 k^{2}} A^{*}\right] v_{1} v_{2} e^{-i x} \\
& +\left[\frac{2(1-2 k+2 c)}{1+4 c-4 k^{2}} A-\frac{4 c}{1+4 c-4 k^{2}} B\right] v_{1} v_{2} e^{i x} \\
& +\left[\frac{(2-2 k) C}{4+4 c-4 k^{2}}+\frac{c(C-D)}{4+4 c-4 k^{2}}\right] v_{1}^{2} e^{2 i x} .
\end{aligned}
$$

Here, the coefficients $A, B, C$ and $D$ are given in the Appendix A.

Substituting the condensate function $\psi(x) \approx \psi^{(0)}(x)+$ $\psi^{(1)}(x)+\psi^{(2)}(x)$ into the Hamiltonian (2.3), we derive the energy of the BEC and calculate the effective mass $m^{*}$ and the compressibility $\kappa$ from equations (3.3) and (3.4), respectively. The results are

$$
\begin{aligned}
\frac{1}{\kappa}=c & +\frac{8 c}{(1+4 c)^{3}} v_{1}^{2}+\frac{c}{2(1+c)^{3}} v_{2}^{2} \\
& -\frac{27 c}{(1+c)^{3}(1+4 c)^{4}} v_{1}^{2} v_{2} \cos (\phi),
\end{aligned}
$$

and

$$
\begin{aligned}
\frac{1}{m^{*}}=1 & -\frac{8}{(1+4 c)^{2}} v_{1}^{2}-\frac{1}{2(1+c)^{2}} v_{2}^{2} \\
& -\frac{15}{(1+c)^{2}(1+4 c)^{3}} v_{1}^{2} v_{2} \cos (\phi) .
\end{aligned}
$$

The sound speed in equation (3.2) is therefore readily derived as

$$
\begin{aligned}
c_{s} & =\sqrt{c}\left(1-\frac{16 c}{(1+4 c)^{3}} v_{1}^{2}-\frac{1+2 c}{4(1+c)^{3}} v_{2}^{2}\right) \\
& -\frac{27+15(1+c)(1+4 c)}{(1+c)^{3}(1+4 c)^{4}} v_{1}^{2} v_{2} \cos (\phi) .
\end{aligned}
$$

Equation (3.10) is one of the key results of this paper. When $v_{2}=0$, equation (3.10) can recover the corresponding result in reference [26] and agrees with the conclusion that the sound velocity of a BEC in an OL of $v_{1} \cos x$ decreases monotonically with $v_{1}$, as it should be. In the presence of $v_{2}$ and $\phi \neq 0$, the situation is very different, where the sound velocity can instead increase with increasing $v_{2}$ for $\phi=\pi$. This is shown in Fig. $3 \mathrm{~d}$, which reflects a greater influence from $\kappa$ than $m^{*}$ on the sound velocity. Such behavior can be expected from analysis of equation (3.10), where the last two terms in the brackets are definitely negative, whereas the terms in the second line can be either positive or negative depending on $\phi$. In particular, if we choose $\cos \phi<0$, the last term in the brackets of equation (3.10) appears at the second order of $v_{2}$, whereas in comparison the last term of equation (3.10) emerges at the first-order. Consequently, in the weak potential limit the sound speed must first increase with increasing $v_{2}$. This observation can be intuitively summarized within following picture: the variation of relative phase $\phi$ (see Fig. 1b) can lead to a more tightly squeezed condensate, which will greatly decrease the value of the $\kappa$ and give rise to an increased sound velocity.

\section{Tight-binding regime}

We now turn to the tight-binding regime with $v_{2} \ll v_{1}$. In this case, the lattice potential $v_{2} \cos (2 x+\phi)$ can be considered as a perturbation, while the unperturbed system consists of a quasi-1D BEC tightly confined in an optical lattice $v_{1} \cos (x)$. The unperturbed tightly confined BEC system can be well described using the tightbinding model [60], where the condensate wavefunction $\psi(x)$ can be written as a superposition form as [5]

$$
\psi(x)=\sum_{n} \varphi_{n}(x) \psi_{n}
$$

where $n$ is the site number of the lattice $v_{1} \cos (x), \varphi_{n}(x)$ is the condensate wavefunction in the $n$th well with $\varphi_{n}(x)=\varphi_{0}(x+n d)$ and $\psi_{n}$ is the corresponding expansion coefficients. In the presence of additional lattice potential $v_{2} \cos (2 x+\phi)$, for $v_{2} \ll v_{1}$, we expect that the effect of the $v_{2}$ lattice is to cause small modifications to the condensate function $\psi(x)$ in (3.11). We therefore use a variational approach and take equation (3.11) as a variational ansatz for the condensate function $\psi(x)$ in the presence of the $V_{2}$ lattice, with $\psi_{n}$ being the variational parameters. Substituting the variational ansatz (3.11) into equation (2.3), we recast the Hamiltonian $H_{1 D}$ as

$$
H=-J \sum_{n}\left(\psi_{n}^{*} \psi_{n+1}+\psi_{n+1}^{*} \psi_{n}\right)+\frac{U}{2} \sum_{n}\left|\psi_{n}\right|^{4},
$$

with the hopping amplitude

$$
J=-\frac{1}{(2 \pi)} \int d x\left[\frac{1}{2}\left(\partial_{x} \varphi_{n} \cdot \partial_{x} \varphi_{n+1}\right)+\varphi_{n} V_{\mathrm{OSL}} \varphi_{n+1}\right]
$$

and the on-site interaction

$$
U=\frac{c}{(2 \pi)} \int d x \varphi_{n}^{4}
$$

It follows from equations (3.12)-(3.14) that the effect of a weak $V_{2}$ lattice is to modify the hopping amplitude $J$ and the on-site interaction $U$.

The ground state of Hamiltonian (3.12) is a constant wave function $\psi_{n}=1$. As a standard procedure [26], its 
excitation energy is given by $\epsilon\left(q_{x}\right)=2\left|\sin \left(q_{x} \pi\right)\right| \sqrt{2 J_{x} U}$. We now calculate $m^{*}$ and $\kappa$ in terms of $J$ and $U$ [5], respectively. First, in order to derive the compressibility $\kappa$ in equation (3.4), we use a Gaussian form $\varphi_{n}(x)=\left(\pi^{1 / 4} \sigma_{x}^{1 / 2}\right) \exp \left[-x^{2} / 2 \sigma_{x}^{2}\right]$ for the condensate function $\varphi_{n}(x)$ in calculating the $U$ in equation (3.14), where the width $\sigma_{x}$ of the condensate function is to be determined variationally. The result isgiven by

$$
\kappa=\frac{1+16\left[v_{1}+4 v_{2} \cos (\phi)\right]^{1 / 2}}{\sqrt{16 \pi} c\left[v_{1}+4 v_{2} \cos (\phi)\right]^{3 / 4}} .
$$

It follows from equation (3.15) that the compressibility $\kappa$ in the tight-binding limit decreases with $v_{2}$ in a nonexponential form, similarly as the weak potential limit. Next, we derive the effective mass in equation (3.3) by using similar methods to calculate $J$ as in reference [27]. The resulting expression is written as

$$
\begin{aligned}
\frac{m}{m^{*}}= & \frac{1}{4}\left[\left(d / \sigma_{x}\right)^{4}-2\left(d / \sigma_{x}\right)^{2}\right] \exp \left[-\left(d / 2 \sigma_{x}\right)^{2}\right] \\
- & 8 \pi^{2} v_{1} \exp \left[-\left(d / 2 \sigma_{x}\right)^{2}-\pi^{2}\left(\sigma_{x} / d\right)^{2}\right] \\
& +8 \pi^{2} v_{2} \cos (\phi) \exp \left[-\left(d / 2 \sigma_{x}\right)^{2}-4 \pi^{2}\left(\sigma_{x} / d\right)^{2}\right] \\
- & \frac{16 c \pi^{2} d}{\sqrt{2 \pi} \sigma_{x}} \exp \left[-\left(\sqrt{3} d / 2 \sqrt{2} \sigma_{x}\right)^{2}\right] .
\end{aligned}
$$

Note that equation (3.16) is explicitly a single-particle result and is only valid for weak interactions. Plugging equations (3.15) and (3.16) into equation (3.2), the analytical expression of sound velocity in the tight-binding limit can be obtained, which is too complex to write down here.

We stress that the tight-binding model here is restricted to weak $V_{2} \ll V_{1}$ as is shown in Figure $1 \mathrm{~b}$, where the substructure in each well can be safely neglected. For strong $V_{2}$, the substructure has to be taken into account and the tight-binding treatment in reference [46] is a reliable method.

\section{Numerical methods in the whole regime}

In the previous section, we have considered the case where the intensity $V_{2}$ is weak, while the intensity $V_{1}$ is either small $\left(V_{1} \sim V_{2}\right)$ or much more stronger $\left(v_{1} \gg\right.$ $\left.v_{2}\right)$. Our analytical result already reveals new features on the sound velocity caused by the superlattice, when compared to the monochromatic optical lattice potential. In this section, we will address the problem of calculating the sound velocity in a more broader regimes using the numerical approach.

The key step of our approach consists in numerically solving the GPE equation (2.5) based on the Bloch expansion in equation (2.7) for the condensate function $\psi(x)$ (the expansion is cut off at a certain number $m=N$ in the numerical calculations). After the Bloch expansion coefficients $a_{m}$ are found and the condensate function
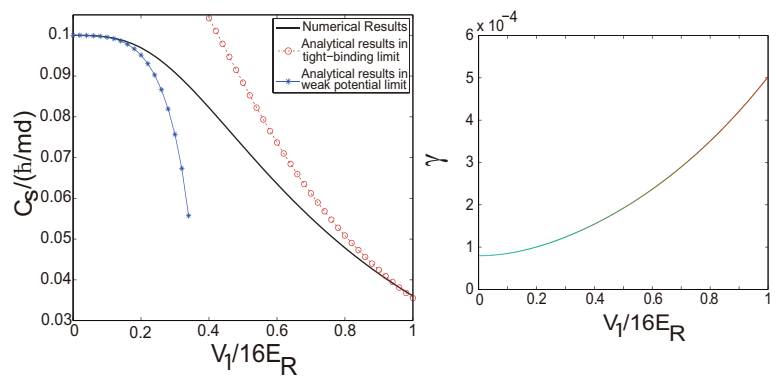

FIG. 2. (Color online) Left panel: sound velocity $c_{s}$ in unit of $\hbar / m d$ for a BEC in an OSL via the lattice strength of $v_{1}=V_{1} / 16 E_{R}$. The numerical results are denoted by the solid lines, analytical results from equation (3.10) in weak potential limit by stars, and analytical tight-binding results by circles obtained by plugging equations (3.15) and (3.16) into equation (3.2). Right panel: the exponent $\gamma$ of the offdiagonal one-body density matrix defined in equation (2.11) via the lattice strength of $v_{1}=V_{1} / 16 E_{R}$, which justifies the validity of the mean-field theoty. The parameters are given by $g n_{0} d=c \times 8 E_{R}, V_{2}=v_{2} \times 16 E_{R}=0.48 E_{R}$, and $\phi=$ $2 \theta+\pi=0$, respectively.

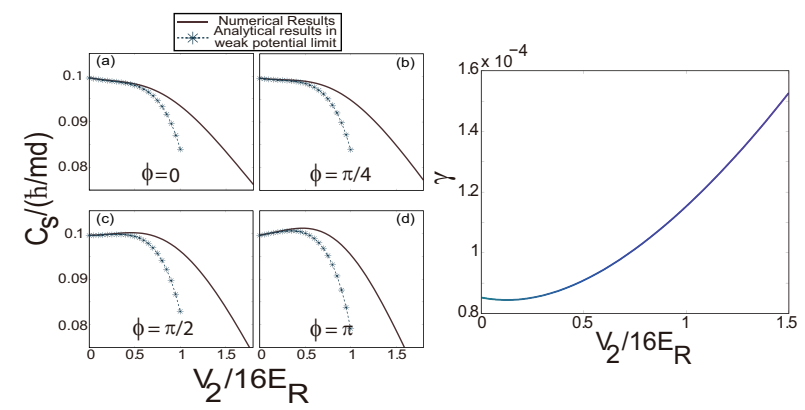

FIG. 3. (Color online) Left panel: sound velocity $c_{s}$ in unit of $\hbar / m d$ for a BEC in an OSL via the lattice strength of $v_{2}=$ $V_{2} / 16 E_{R}$. The numerical results are denoted by the solid lines and analytical results from equation (3.10) in weak potential limit by stars. (a) $\phi=2 \theta+\pi=0$; (b) $\phi=2 \theta+\pi=\pi / 4$; (c) $\phi=2 \theta+\pi=\pi / 2$; (d) $\phi=2 \theta+\pi=\pi$. Right panel: the exponent $\gamma$ of off-diagonal one-body density matrix defined in equation (2.11) via the lattice strength of $v_{2}=V_{2} / 16 E_{R}$ with $\phi=2 \theta+\pi=0$, which justifies the validity of the meanfield theory. The parameters are given by $g n_{0} d=c \times 8 E_{R}=$ $0.08 E_{R}$ and $V_{1}=v_{1} \times E_{R}=1.6 E_{R}$, respectively.

$\psi(x)$ are obtained, we accordingly calculate the energy $E_{k}$ from equation (2.3), which will lead to the derivation of the compressibility $\kappa$, the effective mass $m^{*}$ and the sound velocity $c_{s}$ thereof. In order to comprehensively reveal the effect of superlattice, including the strength of the individual constituting lattice $\left(v_{1}\right.$ and $\left.v_{2}\right)$, and the relative phase $\phi$, on the sound velocity, we have considered three cases for numerical analysis:

(i) In the first step (see Fig. 2), we fix the intensity $v_{2}=V_{2} / 16 E_{R}$ at a small value $\left(V_{2} \sim 0.5 E_{R}\right)$ as well as fixing the relative phase $\phi$, and scan the sound velocity $c_{s}$ as a function of $v_{1}=V_{1} / 16 E_{R}$. As is shown in the 


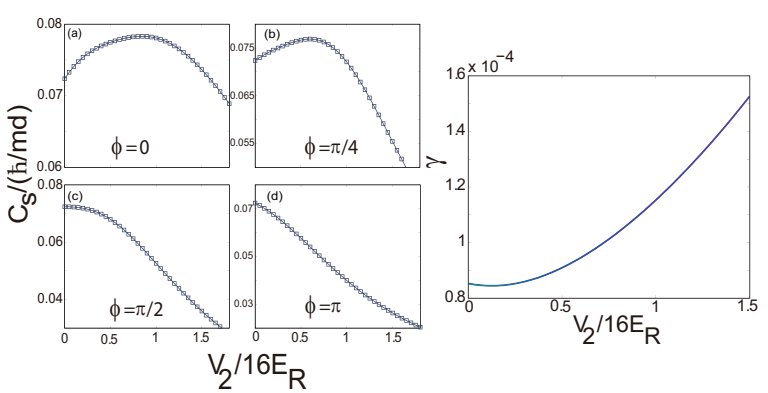

FIG. 4. (Color online) Left panel: sound velocity $c_{s}$ in unit of $\hbar / m d$ for a BEC in an OSL via the OSL strength of $v_{2}=$ $V_{2} / 16 E_{R}$. (a) $\phi=2 \theta+\pi=0$; (b) $\phi=2 \theta+\pi=\pi / 4$; (c) $\phi=2 \theta+\pi=\pi / 2$; (d) $\phi=2 \theta+\pi=\pi$. Right panel: the exponent $\gamma$ of off-diagonal one-body density matrix defined in equation (2.11) via the OSL strength of $v_{2}=V_{2} / 16 E_{R}$, which justifies the validity of the mean-field theory. The parameters are given by $g n_{0} d=c \times 8 E_{R}=0.08 E_{R}$ and $V_{1}=v_{1} \times 16 E_{R}=$ $1.6 E_{R}$, respectively.

left panel of Figure 2, the sound velocity $c_{s}$ decreases monotonically with increasing $V_{1}$ as expected. For small $V_{1} \sim V_{2}$ (weak potential limit) and for $V_{1} \gg V_{2}$ (tightbinding limit), we see that the numerical result agrees well with our analytical results as they should be. Moreover, we have checked that our numerical results in limit of $V_{2}=0$ can recover the corresponding one in references $[19,26]$.

(ii) In the second step (see Fig. 3), we fix $v_{1}=$ $V_{1} / 16 E_{R}$ at a small value $\left(V_{1} \sim 1.6 E_{R}\right.$ in Figure 3$)$, and scan the sound velocity $c_{s}$ as a function of $v_{2}=V_{2} / 16 E_{R}$ for various choice of $\phi$. As is shown, in the upper two figures of Figure 3 ( $\phi=0, \pi / 4$, respectively), the sound velocity still shows an overall decrease with increasing $V_{2}$. Whereas, when $\phi=\pi / 2, \pi$, it is clearly shown that the sound velocity $c_{s}$ firstly increases and then decreases with increasing $V_{2}$. The numerical results depicted in Figure 3 can be understood using equation (3.10): the last term in equation (3.10) can be positive or negative depending on $\phi$, whereas the last two terms in the bracket of equation (3.10) are always negative. Naively, one would expect that the sound velocity either decreases monotonically with $V_{2}$ for the case of $\cos \phi>0$ (see Figs. 3a and 3b) or may develop a maximum for $\cos \phi<0$ (see Figs. $3 \mathrm{c}$ and $3 \mathrm{~d}$ ). This is exactly what we have seen in Figure 3. However, for large $V_{2}$ in Fig. 3, a marked discrepancy emerges between the tight-binding results and the numerical one. This can be traced back to the fact that the substructures in each lattice well have been neglected in the tight-binding treatment, which nevertheless plays increasingly important role on determining the sound speed for larger $V_{2}$.

(iii) To further highlight the interplay between the two lattices with different periods, in the third step (see Fig. 4), we choose a strong intensity $v_{1}=V_{1} / 16 E_{R}$ $\left(V_{1}=9.6 E_{R}\right.$ in Fig. 4), and scan the sound velocity as a function of $V_{2}$ for various choice of $\phi$. As shown in
Figures $4 \mathrm{a}$ and $4 \mathrm{~b}$, it turns out that the sound velocity achieves a maximum value for $\phi=0, \pi / 4$. These results in Figures $4 \mathrm{a}$ and $4 \mathrm{~b}$ are not conflict with equation (3.10), which suggests that a maximum of sound velocity can be only developed for $\phi=0, \pi / 4$, because equation (3.10) becomes to be invalid in the case of strong $V_{1}$. We attribute the explanation for Figures $4 \mathrm{a}$ and $4 \mathrm{~b}$ to the competition between the compressibility and effective mass through equation (3.2) and such competition is supposed to manifest itself quite differently with respect to the various choices of OSL's parameters, resulting in the difference between Figures 3 and 4 . On the other hand, motivated by reference [61], another possible intuitive explanation for sound velocity in Figures 3 and 4 can be carried out in terms of the effective dimensionality seen by a BEC. The lattice of $V_{2}$ consists of the $x$-direction of our system, while the introduction of the strong strength $V_{1}$ plays the role of squeezing a BEC reminiscent of the other spatial dimensionality of the model system. Therefore, we can reasonably view our system as an effective 2D BEC loaded in a monochromatic OL with the role of the dimensionality taken by the $V_{1}$ from the view of they both squeezing the condensate. Figures 3 and 4 become to be understood in the framework of reference [26], i.e. sound velocity of an optically-trapped BEC in 2D may develop a maximum with the increasing lattice strength. More importantly, in Figure 4, the maximum of sound velocity can exceed that for $V_{2}=0$ with the same $V_{1}$ by $10 \%$. Moreover, the maximum falls into the superfluid regimes, and should be observable within the current experimental conditions. Finally, to make our investigation of sound velocity more completely, we have plotted Figure 5 with the fixed value of $\phi=0$, which shows how the sound velocity varies via both the $V_{1} / 16 E_{R}$ and $V_{2} / E_{R}$.

Meanwhile, as shown by the right panel of Figures 2-4, we have monitored that the exponent $\gamma$ of off-diagonal one-body density matrix is always much smaller than 1 , justifying the calculations of sound speed in the framework of the mean-field theory based on GPE. Note that our study on the sound propagation has been done based on the GPE which consists of ignoring the quantum fluctuations and temperature effects. To study the effects of finite temperature and fluctuations, particularly near the transition point of superfluid and Mott insulator, one has to use other theories [53].

\section{POSSIBLE EXPERIMENTAL SCENARIOS AND CONCLUSION}

The present model on the sound velocity of a quasi1D BEC in an OSL is based on GPE and highlights the competition between the compressibility $\kappa$ and the effective mass $m^{*}$. We have shown that the sound velocity is characterized by four parameters: the effective interatomic interaction $c$, the lattice intensities $V_{1(2)}$ and the relative phase $\phi$ between the fundamental lattice and the 


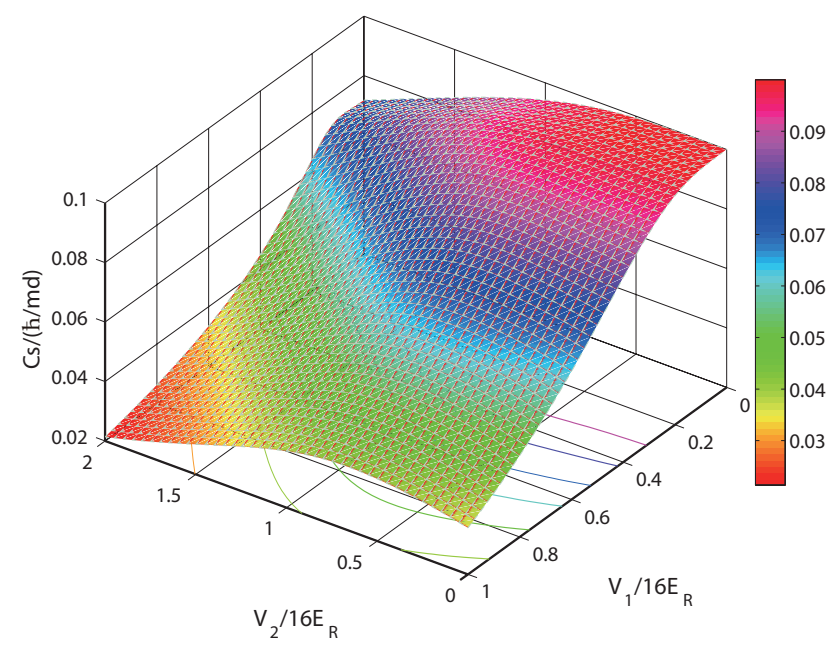

FIG. 5. (Color online) Sound velocity $c_{s}$ in unit of $\hbar / m d$ for a BEC in an OSL via both the lattice strengths of $v_{1}=$ $V_{1} / 16 E_{R}$ and $v_{2}=V_{2} / 16 E_{R}$. The parameters are given by $g n_{0} d=c \times 8 E_{R}=0.08 E_{R}$ and $\phi=2 \theta+\pi=0$.

double-period lattice. All these quantities are experimentally controllable using state-of-the-art technologies. The interatomic interaction can be controlled in a very versatile manner via the technology of Feshbach resonances [62]. In typical experiments to date [5], the values of $c$ range from 0.01 to $0.1 E_{R}$. The strength of both $V_{1}$ and $V_{2}$ an be tuned from $0 E_{R}$ to $32 E_{R}$ almost at will. Phase control [46] between the two standing wave fields allows $\phi$ to scan the whole range $[0,2 \pi]$.

Central to testing the validity of the prediction in this article is the experimental ability to measure the sound velocity in a BEC. The experimental approaches so far to sound velocity are based on analyzing the linear response of a fluid to an external velocity boost. The key quantity to measure in these approaches is the dynamic structure factor of the model system $[3,4]$. The speed of sound of a $\mathrm{BEC}$ in an OL may be measured with a similar technique as was used in references $[9,63]$. Another option is to employ Bragg spectroscopy $[10,11,64]$ to the excitation spectrum. The sound velocity can be extracted from the slope of the linear part of the excitation spectrum.

Another difficulty may arise from the typical application of an external harmonic trap with the frequency of $\omega$ in the BEC experiments, which will lead to the discrete spectrum due to the finite size of the trapped BEC [65]. The detailed concern is as follows: our definition of sound velocity is based on equation (3.2), where the presence of the OL has been accounted for through renormalizing compressibility $\kappa$ and the effective mass $m^{*}$. As a result, despite the presence of the lattice, one can study the sound velocity of a BEC using equation (3.2) as if the space is homogeneous. In such, with the considerations of the harmonic trap, the condensate wave-function is non-vanishing over a size of the $l=(\sqrt{2} / \pi) c_{s} / \omega$ in the
Thomas-Fermi approximation. As a compression, the frequencies of the lowest collective excitations are proportional to $c_{s} / l \propto \omega$, being simply proportional to the trapping frequencies, which do not yield direct information of the sound velocity. Therefore, in order to observe the sound speed, one must excite the perturbation much smaller than the size of the condensate by ruling out the collective excitation of the model system.

On overcoming the preceding two difficulties, the experimental realization of our scenario amounts to controlling four parameters whose interplay underlies the physics of this work. Therefore, the phenomena discussed in this article should be observable within the current experimental capability, which would constitute an important step in understanding the effect of an OSL on the superfluidity of a BEC.

In summary, we have studied analytically and numerically the sound speed of a 1D BEC in an OSL. Our results show that the interplay between two constituting lattices that have different periods can significantly influence the sound velocity of the BEC system. In particular, unusual behavior of sound propagation in an OSL compared to the case in an OL at $1 \mathrm{D}$ has been found, i.e. the sound speed can first increase and then decrease as the lattice strength $V_{2}$ increases. Such behavior can be understood using our analytical results for weak lattices. As a consequence, an experimentalist can, in principle, engineer the rich behavior of the sound speed by altering OSL's parameters, which can find the direct applications for manipulating system bath coupling in driven open system with atoms.

\section{ACKNOWLEDGMENTS}

We thank Ying Hu and Biao Wu for helpful discussions and Teng Yang for carefully reading our manuscript. This work is supported by the NSF of China (Grants Nos. 11004200 and 11274315).

\section{Appendix A: Preliminary notations}

The explicit expressions of $A, B, C$, and $D$ in equation (3.7) read as follows respectively,

$$
\begin{aligned}
& A=\frac{1}{8} \frac{\left(4 k^{2}-7\right)(k+2 c+2)+9(2 c+1)}{\left(1+c-k^{2}\right)\left(4 k^{2}-4 c-1\right)} e^{i \phi} \\
& B=-\frac{1}{8} \frac{\left(4 k^{2}-7\right)(k-2 c-2)-9(2 c+1)}{\left(1+c-k^{2}\right)\left(4 k^{2}-4 c-1\right)} e^{i \phi} \\
& C=\frac{1}{2} \frac{\left(4 k^{2}-1\right)(2 k+2 c-1)}{\left(4 k^{2}-4 c-1\right)^{2}} \\
& D=-\frac{1}{2} \frac{\left(4 k^{2}-1\right)(2 k-2 c+1)}{\left(4 k^{2}-4 c-1\right)^{2}}
\end{aligned}
$$


[1] F. Dalfovo, S. Giorgini, L. P. Pitaevskii, and S. Stringari, Rev. Mod. Phys. 71, 463 (1999).

[2] L. Pitaevskii and S. Stringari, Bose-Einstein Condensation (Clarendon Press, Oxford, 2003).

[3] D. Pines and P. Noziéres, The Theory of Quantum Liquids (Benjamin, New York, 1966 ), Vol. I

[4] P. Noziéres and D. Pines, The Theory of Quatum Liquids (Addison-Wesley, Reading, MA, 1990 ), Vol. II.

[5] I. Bloch, J. Dalibard, and W. Zwerger, Rev. Mod. Phys. 80, 885 (2008).

[6] M. Lewenstein, A. Sanpera, and V. Ahufinger, Ultracold Atoms in Optical Lattices: Simulating quantum manybody systems, Oxford University Press, Oxford, 2012.

[7] M. R. Andrews, D. M. Kurn, H. J. Miesner, D. S. Durfee, C. G. Townsend, S. Inouye, and W. Ketterle, Phys. Rev. Lett. 79, 553 (1997).

[8] M. R. Andrews, D. M. Stamper-Kurn, H. J. Miesner, D. S. Durfee, C. G. Townsend, S. Inouye, and W. Ketterle, Phys. Rev. Lett. 80, 2967 (E) (1998).

[9] C. Raman, M. Kohl, R. Onofrio, D.S. Durfee, C.E. Kuklewicz, Z. Hadzibabic, W. Ketterle, Phys. Rev. Lett. 83, $2502(1999)$

[10] X. Du, S. Wan, E. Yesilada, C. Ryu, D. J. Heinzen, Z. Liang, and B. Wu, New. J. Phys. 12, 083025 (2010).

[11] P. T. Ernst, S. Götze, J. S. Krauser, K. Pyka, D. S. Lüann, D. Pfannkuche, and K. Sengstock, Nat. Phys. 6, 56 (2009).

[12] P. Öhberg, E. L. Surkov, I. Tittonen, S. Stenholm, M. Wilkens, and G. V. Shlyapnikov, Phys. Rev. A 56, R3346 (1997).

[13] E. Zaremba, Phys. Rev. A 57, 518 (1998).

[14] G. M. Kavoulakis and C. J. Pethick, Phys. Rev. A 58, 1563 (1998).

[15] S. Stringari, Phys. Rev. A 58, 2385 (1998).

[16] P. O. Fedichev and G. V. Shlyapnikov, Phys. Rev. A 63, 045601 (2001).

[17] B. Damski, Phys. Rev. A 69, 043610 (2004).

[18] K. Berg-Sorensen and K. Molmer, Phys. Rev. A 58, 1480 (1998).

[19] M. Krämer, C. Menotti, L. Pitaevskii, and S. Stringari, Eur. Phys. J. D 27, 247 (2003).

[20] C. Menotti, M. Krämer, A. Smerzi, L. Pitaevskii, and S. Stringari, Phys. Rev. A 70, 023609 (2004).

[21] I. Danshita, S. Kurihara, and S. Tsuchiya, Phys. Rev. A 72, 053611 (2005).

[22] J. P. Martikainen and H. T. C. Stoof, Phys. Rev. A 69, 023608 (2004).

[23] M. Krämer, C. Menotti, and M. Modugno, J. Low Temp. Phys. 138, 729 (2005).

[24] E. Taylor and E. Zaremba, Phys. Rev. A 68, 053611 (2003).

[25] D. Boers, C. Weiss, and M. Holthaus, Europhys. Lett. 67, 887 (2004).

[26] Z. X. Liang, X. Dong, Z. D. Zhang, and B. Wu, Phys. Rev. A 78, 023622 (2008).

[27] W. Zwerger, J. Opt. B: Quantum Semiclassical Opt. 5, S9 (2003).

[28] Ying $\mathrm{Hu}$, Zhaoxin Liang, and Bambi Hu, Phys. Rev. A 80, 043629 (2009).

[29] Ying Hu, Zhaoxin Liang, and Bambi Hu, Phys. Rev. A 81, 053621 (2010).
[30] D. Jaksch and P. Zoller, Ann. Phys. 315, 52 (2005).

[31] J. I. Cirac and P. Zoller, Nat. Phys. 8, 264 (2012)

[32] I. Bloch, J. Dalibard, and S. Nascimbéne, Nat. Phys. 8, 267 (2012).

[33] S. Diehl, A. Micheli, A. Kantian, B. Kraus, H. P. Büchler, and P. Zoller, Nat. Phys. 4, 878 (2008).

[34] A. J. Daley, P. O. Fedichev, and P. Zoller, Phys. Rev. A 69, 022306 (2004).

[35] A. Griessner, A. J. Daley, S. R. Clark, D. Jaksch, and P. Zoller, Phys. Rev. Lett. 97, 220403 (2006).

[36] A. Griessner, A.J. Daley, S. R. Clark, D. Jaksch, and P. Zoller, New J. Phys. 9, 44 (2007).

[37] P. Windpassinger and K. Sengstock, Rep. Prog. Phys. 76, 086401 (2013).

[38] S. Peil, J.V. Porto, B.L. Tolra, J.M. Obrecht, B.E. King, M. Subbotin, S.L. Rolston, W.D. Phillips, Phys. Rev. A 67, 053403(R) (2003).

[39] M. Weitz, G. Cennini, G. Ritt, and C. Geckeler, Phys. Rev. A 70, 043414 (2004).

[40] G. Ritt, C. Geckeler, T. Salger, G. Cennini, and M. Weitz, Phys. Rev. A 74, 063622 (2006).

[41] T. Salger, C. Geckeler, S. Kling, and M. Weitz, Phys. Rev. Lett. 99, 190405 (2007).

[42] T. Salger, C. Geckeler, S. Kling, and M. Weitz, Phys. Rev. Lett. 107, 240401 (2011).

[43] A.B. Bhattacherjee, J. Phys. B 40, 143 (2007).

[44] G. Roati, C. D'Errico, L. Fallani, M. Fattori, C. Fort, M. Zaccanti, G. Modugno, M. Modugno, M. Inguscio, Nature 453, 895 (2008).

[45] S. Trotzky, P. Cheinet, S. Foelling, M. Feld, U. Schnorrberger, A.M. Rey, A. Polkovnikov, E.A. Demler, M.D. Lukin, and I. F. Bloch, Science 319, 295 (2008).

[46] M. Atala, M. Aidelsburger, J. T. Barreiro, D. Abanin, T. Kitagawa, E. Demler, and I. Bloch, Nat. Phys. 9, 795 (2013).

[47] S.L. Zhu, Z.D. Wang, Y.H. Chan, L.M. Duan, Phys. Rev. Lett. 110, 075303 (2013).

[48] Z.H. Xu, L.H. Li, S. Chen, Phys. Rev. Lett. 110, 215301 (2013).

[49] J. I. Cirac, P. Maraner, and J. K. Pachos, Phys. Rev. Lett. 105, 190403 (2010).

[50] S. Fölling, S. Trotzky, P. Cheinet, M. Feld, R. Saers, A. Widera, T. Müller, and I. F. Bloch, Nature (London) 448, 1029 (2007).

[51] L. Wang, M. Troyer, and X. Dai, Phys. Rev. Lett. 111, 026802 (2013).

[52] F.S. Cataliotti et al., Science 293, 843 (2001).

[53] D. S. Petrov, G. V. Shlyapnikov, and J. T. M. Walraven, Phys. Rev. Lett. 87, 050404 (2001).

[54] Ying $\mathrm{Hu}$ and Zhaoxin Liang, Phys. Rev. Lett. 107, 110401 (2011).

[55] Ying $\mathrm{Hu}$ and Zhaoxin Liang, Mod. Phys. Lett. B 27, 1330010 (2013).

[56] M. Olshanii, Phys. Rev. Lett. 81, 938 (1998).

[57] C. Menotti and S. Stringari, Phys. Rev. A 66, 043610 (2002).

[58] Biao Wu and Qian Niu, Phys. Rev. A 64, 061603 (2001).

[59] Biao Wu and Qian Niu, New J. Phys. 5, 104 (2003).

[60] A. Smerzi, A. Trombettoni, Phys. Rev. A 68, 023613 (2003).

[61] L.J. Lang, X.M. Cai, S. Chen, Phys. Rev. Lett. 108, 
220401 (2012).

[62] C. Chin, R. Grimm, P. Julienne, and E. Tiesinga, Rev. Mod. Phys. 82, 1225 (2010).

[63] L. A. Sidorenkov, M. K. Tey, R. Grimm, Y. H. Hou, L. Pitaevskii, and S. Stringari, Nature(London), 498, 78 (2013).
[64] L. Chen, W. Li, Z. Chen, Z. Zhang, Z. Liang, J. Low. Temp. Phys. 177, 291 (2014).

[65] L. Salasnich, A. Parola, L. Reatto, Phys. Rev. A 65, 043614 (2002). 